\title{
SUR LES SURFACES DES ZÉROS DES FONCTIONS HOLOMORPHES DANS LES DOMAINES INTÉRIEUREMENT RAMIFIÉS
}

\author{
Par \\ Masahito TAKASE \\ (Reçu le 9 mars, 1982) \\ (Révisé le 17 septembre, 1982)
}

\section{Introduction.}

$1^{\circ}$. En 1951, K. Oka [4] a indiqué déjà le fait très remarquable que voici: En général, une surface caractéristique sur un domaine intérieurement ramifié ne peut pas se représenter même localement comme l'ensemble des zéros d'une seule fonction holomor phe sur le domaine. Quelques années après, H. Grauert$\mathrm{R}$. Remmert [1] ont donné réellement l'exemple d'un domaine intérieurement ramifié et une surface caractéristique sur le domaine au caractère précité. Nous ne pouvons jamais voir ce phénomène dans les domaines sans point critique intérieur. Cet exemple montre qu'il y a une restriction à laquelle sont soumises les surfaces des zéros des fonctions holomorphes sur les domaines intérieurement ramifiés. Nous allons rechercher dans le présent mémoire et celui qui suit les caractères de cette restriction; c'est notre problème fondamental. Ces recherches, je pense, donnent le base de la théorie locale des domaines intérieurement ramifiés.

Dans le présent mémoire, nous allons rechercher en détail l'exemple donné par H. Grauert-R. Remmert [1]; et dans le mémoire qui suit, nous allons rechercher notre problème fondamental d'une manière générale.

$2^{\circ}$. Expliquons l'exemple de H. Grauert-R. Remmert [1]. Dans l'espace $C^{2 k}(z, w)$ des $2 k$ variables complexes $z_{1}, w_{1}, z_{2}, \ldots, z_{k}, w_{k}$ dont $k \geqq 2$, considérons la variété caractéristique $Y$ donnée par les équations

$$
\frac{z_{1}}{w_{1}}=\frac{z_{2}}{w_{2}}=\cdots=\frac{z_{k}}{w_{k}}
$$

et l'application $\Phi$ de $Y$ dans l'espace $C^{k+1}(v)$ des $(k+1)$ variables complexes 
$v_{0}, v_{1}, \ldots, v_{k}$ donnée par

$$
\begin{gathered}
v_{0}=z_{1}+z_{2}+\cdots+z_{k} \\
v_{1}=z_{1}+a_{1} w_{1} \\
\\
\cdots \\
v_{k}=z_{k}+a_{k} w_{k}
\end{gathered}
$$

où $a_{i}(i=1,2, \ldots, k)$ sont des constantes complexes telles que $\left|a_{i}\right|=1(i=1,2, \ldots, k)$ et $a_{i} \neq a_{j}(i \neq j)$. Alors, le triple $\xi=\left(Y, \Phi, \mathbf{C}^{k+1}(v)\right)$ est un revêtement analytiquement ramifié de $C^{k+1}(v)$ à $k$ feuilles. Pour l'origine $O$ de l'espace $C^{k+1}(v)$, la fibre $\Phi^{-1}(O)$ se compose d'un seul point que nous designons par $y_{0} ; y_{0}$ est un point de ramification qui n'est pas uniformisable.

Dans ce revêtement $\xi$, considérons la famille $\{E(s)\}$ composée des surfaces caractéristiques irréductibles $E(s)$ données par les équations

$$
\frac{z_{1}}{w_{1}}=\frac{z_{2}}{w_{2}}=\cdots=\frac{z_{k}}{w_{k}}=s
$$

où $s$ est un paramètre qui parcourt partout sur la sphère de Riemann $\boldsymbol{P}(s)$. Alors, on a

$$
Y=\underset{s \in \mathbf{P}(s)}{\bigcup} E(s) ;
$$

et pour deux paramètres $s, s^{\prime}$ où $s \neq s^{\prime}$, on a toujours

$$
E(s) \cap E\left(s^{\prime}\right)=\left\{y_{0}\right\} .
$$

Dans ces circonstances, H. Grauert-R. Remmert [1] ont démontré que toute surface caractéristique $E(s)$ ne peut pas se représenter dans aucun voisinage de $y_{0}$ comme l'ensemble des zéros communs de l fonctions holomorphes où $l<k$.

D'autre part, il $y$ a certainement une surface caractéristique irréductible $T$ dans un voisinage de $y_{0}$ qui peut se représenter par une seule fonction holomorphe. Par suite, je trouve naturel qu'il $y$ ait une différence entre $E(s)$ et $T$; c'est un des buts de nos recherches. Nous donnons une condition suffisante pour que, dans les domaines à deux feuilles, une surface caractéristique irréductible peut se représenter comme l'ensemble des zéros d'une seule fonction holomorphe; et nous mentionnons que, dans l'example $\xi$ de H. Grauert-R. Remmert [1], les circonstances de distribution des composantes irréductibles d'une fonction holomorphe irréductible ne sont pas arbitraires. 
Nous remarquons finalemant qu'en général l'ordre de zéro des fonctions holomorphes sur les domaines intérieurement ramifiés ne sont pas arbitraires.

Dans l'exemple $\xi$ de H. Grauert-R. Remmert [1], nous considérons pour plus de brièveté le cas où $k=2$; et posons $a_{1}=1, a_{2}=-1$; alors, on a un revêtement analytiquement ramifié $\xi=\left(Y, \Phi, C^{3}(v)\right)$ de l'espace $C^{3}(v)$ à deux feuilles; nous l'appellerons dans la suivante simplement l'exemple de Grauert-Remmert.

\section{Tables des matières}

1. Surfaces des zéros des fonctions holomorphes sur les revêtement analytiquement ramifiés à deux feuilles.

2. Surfaces des zéros des fonctions holomorphes sur la surface de Riemann de la fonction algébrique $\sqrt{D(x, y)}$ où $D(x, y)$ désigne la forme quadratique $D(x, y)=A x^{2}+B x y+C y^{2}\left(B^{2}-4 A C \neq 0\right)$ de deux variables complexes $x, y$.

3. Deux familles $\{E(s)\}$ et $\{F(s)\}$ des surfaces caractéristiques qui engendrent l'exemple de Grauert-Remmert $\xi=\left(Y, \Phi, C^{3}(v)\right)$.

4. Ordres de zéro des fonctions holomorphes sur les domaines intérieurement ramifiés.

\section{Surfaces des zéros des fonctions holomorphes sur les revêtement analyt- iquement ramifié à deux feuilles.}

$1^{\circ}$. Soient $G$ un domaine dans l'espace $\boldsymbol{C}^{n}(x)$ des $n$ variables complexes $x_{1}, x_{2}, \ldots, x_{n}$, et $\eta=(Z, \Psi, G)$ un revêtement analytiquement ramifié de $G$ aux deux feuilles. Supposons que, pour un point (a) de $G$, la fibre $\Psi^{-1}(a)$ se compose d'un seul point de ramification; désignons-le par $z_{0}$. Dans la suivante, nous nous trouvons toujours dans un voisinage de $z_{0}$.

Grâce à H. Grauert-R. Remmert [2] ou R. Kawai [3], reprenant G suffisamment petit s'il est nécessaire, il $y$ a une fonction holomorphe $h$ sur $\eta$ qui sépare les feuilles de $\eta$ par ses éléments de Taylor; c'est-à-dire, $h$ a les éléments de Taylor différents pour toute paire de points différents dans $\eta$. Puisque $\eta$ est à deux feuilles, il $y$ a un polynôme de Weierstrass du deuxième degré

$$
R(w)=w^{2}+p(x) \cdot w+q(x),
$$

où $p(x), q(x)$ désignent des fonctions holomorphes sur $G$, tel que $R(h)$ s'annule identiquement sur $\eta$. Désignons par $S$ la surface critique de $\eta ; \Psi(S)$ est alors 
une surface caractéristique dans $G$; reprenant $G$ suffisamment petit s'il est nécessaire, on peut supposer qu'il $y$ ait une équation minimum de $\Psi(S)$; désignons-la par $D(x)=0$ où $D(x)$ désigne une fonction holomorphe sur $G$. Puisque $h$ est une fonction sur $\eta$, le discriminant $\{p(x)\}^{2}-4 q(x)$ du polynôme $R(w)$ est divisible par $D(x)$ dans $G$; on peut écrire par suite

$$
\{p(x)\}^{2}-4 q(x)=l(x) \cdot\{D(x)\}^{j}
$$

où $j$ est un entier positif, et $l(x)$ désigne une fonction holomorphe sur $G$ telle qu'elle ne soit pas divisible par $D(x)$ : et de plus on peut écrire

$$
l(x)=\{m(x)\}^{2},
$$

$m(x)$ étant une fonction holomorphe sur $G$, puisque $h$ est une fonction holomorphe sur $\eta$ et que l'équation minimum de $\Psi(S)$ est $D(x)=0 ; j$ est impair, puisque $h$ a les éléments de Taylor différents pour toute paire de points différents dans $\eta$. Par suite, on peut poser $j=2 k+1$ où $k$ est un entier positif ou nul. Alors:

$$
\{p(x)\}^{2}-4 q(x)=\left[m(x) \cdot\{D(x)\}^{k}\right]^{2} \cdot D(x) .
$$

Donc:

$$
h=-\frac{1}{2} p(x)+\frac{1}{2} m(x) \cdot\{D(x)\}^{k} \cdot \sqrt{D(x)}
$$

où $\sqrt{D(x)}$ désigne une fonction algébroïde définie par l'équation du deuxième degré $w^{2}-D(x)=0$.

Nous avons ainsi vu les propositions suivantes:

Proposition 1. $\eta$ est la surface de Riemann de $\sqrt{D(x)}$.

Proposition 2. On peut écrire toute fonction holomorphe $h$ sur $\eta$ en forme de

$$
h=\psi(x)+\phi(x) \cdot \sqrt{D(x)}
$$

où $\psi(x), \phi(x)$ désignent des fonctions holomorphes sur $G$.

Dans la suite, on pose toujours $g=\sqrt{D(x)}$.

$2^{\circ}$. Traçcons une variété caractéristique $A$ dans $G$; désignons par $A_{0}$ l'ensemble composé de tous les points réguliers de $A$; une fonction $u$ sera appelée holomorphe sur $A$ si elle est holomorphe sur $A_{0}$, et si elle est bornée pour $A_{0}$ au 
voisinage d'un point quelconque de $A$. Or, traçons à nouveau une surface caractéristique irréductible $A$ dans $G$ qui passe par le point (a); supposons que $A$ soit différente de $\Psi(S)$; alors, la surface caractéristique $\Psi^{-1}(A)$ sur $\eta$ est ou irréductible ou se décompose en deux composantes irréductibles. Considérons la trace de $D(x)$ sur $A$; et désignons-la par $u ; u$ est alors une fonction holomorphe sur $A ; u$ ne s'annule pas identiquement sur $A$, puisque $A$ est différente de $\Psi(S)$.

Proposition 3. La surface caractéristique $\Psi^{-1}(A)$ sur $\eta$ se décompose en deux composantes si et seulement si l'on peut écrire $u=v^{2}$ où $v$ désigne une fonction holomophe sur $A$.

En effet, supposons d'abord que $\Psi^{-1}(A)$ se décompose en deux composantes irréductibles; désignons-les par $T$, $T^{\prime}$; et désignons par $g_{1}, g_{1}^{\prime}$ les traces de $g$ sur $T, T^{\prime}$ respectivement; $g_{1}, g_{1}^{\prime}$ définissent des fonctions holomorphes sur $A$; désignons-les $\operatorname{par} f, f^{\prime}$ respectivement; on a alors $f=-f^{\prime}$. Grâce à W. F. Osgood, on peut écrire

$$
f=\frac{v^{\prime}}{v}, \quad f^{\prime}=-\frac{v^{\prime}}{v}
$$

où $v, v^{\prime}$ sont des fonctions holomorphes sur $G$. Donc, $v g-v^{\prime}, v g+v^{\prime}$ s'annulent identiquement sur $T, T^{\prime}$ respectivement; par suite, le produit de $v g-v^{\prime}$ par $v g+v^{\prime}$

$$
\left(v g-v^{\prime}\right)\left(v g+v^{\prime}\right)=v^{2} g^{2}-v^{\prime 2}=v^{2} D-v^{\prime 2}
$$

s'annule identiquement sur $\Psi^{-1}(A)=T \cup T^{\prime}$; d'autre part, $v^{2} D-v^{\prime 2}$ s'annule identiquement sur $A$, parce qu'elle est univalente; par suite on a

$$
u=\left(\frac{v^{\prime}}{v}\right)^{2}
$$

Ensuite, nous allons montrer la réciproque. Pour cela, on suppose que l'on puisse écrire $u=v^{2}, v$ étant une fonction holomorphe sur $A$. Grâce à W. F. Osgood, on peut écrire

$$
v=\frac{v_{1}^{\prime}}{v_{1}}
$$

où $v_{1}, v_{1}^{\prime}$ sont des fonctions holomorphes sur $G$. Donc, on a sur $\Psi^{-1}(A)$

$$
0 \equiv g^{2}-D(x)=g^{2}-u=g^{2}-v^{2}
$$




$$
\begin{aligned}
& =g^{2}-\left(\frac{v_{1}^{\prime}}{v_{1}}\right)^{2} \\
& =\frac{1}{v_{1}^{2}}\left(v_{1} g-v_{1}^{\prime}\right)\left(v_{1} g+v_{1}^{\prime}\right) .
\end{aligned}
$$

On a par suite

$$
\left(v_{1} g-v_{1}^{\prime}\right)\left(v_{1} g+v_{1}^{\prime}\right) \equiv 0
$$

sur $\Psi^{-1}(A)$. D'autre part, $v_{1} g-v_{1}^{\prime}, v_{1} g+v_{1}^{\prime}$ ne s'annulent pas identiquement sur $\Psi^{-1}(A)$; donc l'expression précitée montre que $\Psi^{-1}(A)$ se décompose en deux composantes irréductibles $T, T^{\prime}, T, T^{\prime}$ étant des certaines composantes irréductibles des surfaces des zéros de $v_{1} g-v_{1}^{\prime}, v_{1} g+v_{1}^{\prime}$ respectivement.

C.Q.F.D.

$3^{\circ}$. Considérons par exemple l'exemple de Grauert-Remmert. Considérons une fonction holomorphe $z_{1}$, précisément dit, sa trace sur $Y$, sur l'exemple de Grauert-Remmert $\xi=\left(Y, \Phi, C^{3}(v)\right)$. Le polynôme de Weierstrass que cette fonction remplit est

$$
2 w^{2}-\left(2 v_{0}+v_{1}-v_{2}\right) \cdot w+v_{0} v_{1}
$$

le degré de ce polynôme est deux; et ceci est égal au nombre des feuilles de $\xi$; donc la fonction $z_{1}$ a les éléments de Taylor différents pour toute paire de points différents dans $\xi$. Remarquons ici

$$
z_{1}=\frac{1}{4}\left\{2 v_{0}+v_{1}-v_{2}+\sqrt{D(v)}\right\}
$$

où

$$
D(v)=\left(v_{1}-v_{2}\right)^{2}-4 v_{0}\left(v_{1}+v_{2}\right)+4 v_{0}^{2} ;
$$

par suite, $\xi$ est la surface de Riemann d'une fonction algébrique $\sqrt{D(v)}$ définie par l'équation $w^{2}-D(v)=0$.

L'expression $D(v)$ est une forme quadratique par rapport à $v_{0}, v_{1}, v_{2}$. Nous allons chercher dans l'espace $C^{3}(v)$ un plan complexe $L$ passant par l'origine $O$ tel que la trace de $D(v)$ sur $L$ se décompose en un carré d'une expression du premier degré.

Considérons d'abord le cas où l'équation de $L$ est en forme de

$$
v_{0}=a_{1} v_{1}+a_{2} v_{2}
$$


$a_{1}, a_{2}$ étant des constantes complexes; on a alors

$$
\begin{aligned}
D \mid L & =D\left(a_{1} v_{1}+a_{2} v_{2}, v_{1}, v_{2}\right) \\
& =\left(v_{1}-v_{2}\right)^{2}-4\left(a_{1} v_{1}+a_{2} v_{2}\right)\left(v_{1}+v_{2}\right)+4\left(a_{1} v_{1}+a_{2} v_{2}\right)^{2} \\
& =\left(2 a_{1}-1\right)^{2} v_{1}^{2}+2\left(4 a_{1} a_{2}-2 a_{1}-2 a_{2}-1\right) v_{1} v_{2}+\left(2 a_{2}-1\right)^{2} v_{2}^{2}
\end{aligned}
$$

où $D \mid L$ désigne la trace de $D(v)$ sur $L$; celle-ci est une forme quadratique par rapport à $v_{1}, v_{2}$; son discriminant est

$$
\begin{aligned}
& \left(4 a_{1} a_{2}-2 a_{1}-2 a_{2}-1\right)^{2}-\left(2 a_{1}-1\right)^{2}\left(2 a_{2}-1\right)^{2} \\
& \quad=-8\left(2 a_{1} a_{2}-a_{1}-a_{2}\right) ;
\end{aligned}
$$

donc la condition que l'on cherche est

$$
2 a_{1} a_{2}-a_{1}-a_{2}=0 .
$$

Ensuite, dans le cas où l'équation de $L$ est en forme de

$$
v_{1}=b_{0} v_{0}+b_{2} v_{2}
$$

$b_{0}, b_{2}$ étant des constantes complexes, on a de même la condition cherchée

$$
b_{0} b_{2}-b_{0}-2 b_{2}=0 \text {. }
$$

En dernier lieu, dans le cas où l'équation de $L$ est en forme de

$$
v_{2}=c_{0} v_{0}+c_{1} v_{1}
$$

$c_{0}, c_{1}$ étant des constantes complexes, on a de même la condition cherchée

$$
c_{0} c_{1}-c_{0}-2 c_{1}=0 \text {. }
$$

En résumé;

Proposition 4. Dans l'espace $C^{3}(v)$, traçons un plan complexe passant par l'origine $O$

$$
\text { (L) } A v_{0}+B v_{1}+C v_{2}=0
$$

$A, B, C$ étant des constantes complexes telles que $(A, B, C) \neq(0,0,0) . \quad$ La condition nécessaire et suffisante pour que la trace de la forme quadratique $D(v)$ sur $L$ se décompose en un carré d'une expression du premier degré est 


$$
A B+2 B C+A C=0 .
$$

Or, l'équation, $A B+2 B C+A C=0$ représente une courbe algébrique irréductible dans l'espace projectif complexe $\boldsymbol{P}^{2}(A, B, C)$ à deux dimension, où l'on regarde $(A, B, C)$ comme des coordonnées homogèes de $\boldsymbol{P}^{2}(A, B, C)$ : désignons-la par $l$. Par suite, on peut dire comme suit: La famille composée de tous les plans complexes sur lesquels $D(v)$ se décompose en un carré d'une expression du premier degré se paramétrize par la courbe algébrique

$$
\text { (l) } A B+2 B C+A C=0
$$

dans l'espace projectif complexe $\boldsymbol{P}^{2}(A, B, C)$.

On peut voir de plus que cette courbe algébrique $l$ est isomorphe à la sphère de Riemann $\boldsymbol{P}(s)$. En effet, $l$ est l'image de $\boldsymbol{P}(s)$ par le plongement

$$
A=s^{2}-1, B=-s^{2}+s, C=-s^{2}-s
$$

de $\boldsymbol{P}(s)$ dans $\boldsymbol{P}^{2}(A, B, C)$.

Considérons la surface caractéristique $\Phi(E(s))$ dans l'espace $C^{3}(v)$; celle-ci est le plan complexe passant par l'origine $O$ de l'espace $C^{3}(v)$, et se définit par l'équation

$$
\begin{gathered}
\left(s^{2}-1\right) v_{0}-s(s-1) v_{1}-s(s+1) v_{2}=0 \quad(\text { le cas où } s \neq \infty) \\
\left.v_{0}-v_{1}-v_{2}=0 \quad \text { (le cas où } s=\infty\right) .
\end{gathered}
$$

Donc, d'après ce que nous avons vu à l'instant, on peut dire comme suit: $L a$ famille des plans complexes $\{\Phi(E(s))\}$ coincide avec celle que nous avons cherchée dans la proposition 4; par suite, la fibre $\Phi^{-1}(\Phi(E(s)))$ se décompose en dux surfaces caractéristiques irréductibles; l'une est $E(s)$; et désignons l'autre par $F(s)$.

$4^{\circ}$. Traçons une surface caractéristique irréductible $T$ sur $\eta$ qui passe par le point $z_{0}$; supposons que $T$ soit irréductible en $z_{0}$. Désignons par $f(x)=0$ l'équation minimum de $A=\Psi(T)$ dans $G$, où $f(x)$ désigne une fonction holomorphe sur $G ; A$ est une surface caractéristique irréductible passant par le point (a).

Soit $h$ une fonction holomorphe sur $\eta$ dans un voisinage de $z_{0}$ telle que $h\left(z_{0}\right)=0 ; h$ sera appelée réductible en $z_{0}$, si l'on peut écrire $h=h_{1} \cdot h_{2}$, où $h_{1}, h_{2}$ sont des fonctions holomorphes sur $\eta$ dans un voisinage de $z_{0}$ telles que $h_{1}\left(z_{0}\right)=$ $h_{2}\left(z_{0}\right)=0 ; h$ sera appelée irréductible en $z_{0}$, si elle n'est pas réductible en $z_{0}$.

Dans ces circonstances, nous voulons trouver une condition suffisante pour 
Sur les surfaces des zéros des fonctions holomorphes dans les domaines intérieurement ramifiés

qu'il $y$ a une fonction holomorphe sur $\eta$ dans un voisinage de $z_{0}$, s'annulant identiquement sur et seulement sur $T$, et étant irréductible en $z_{0}$.

Considérons d'abord le cas où $\Psi^{-1}(A)=T$ dans un voisinage de $z_{0}$; dans ce cas, il est facile de trouver une telle fonction. En effet, on peut regarder $f(x)$ comme une fonction holomorphe sur $\eta$; et alors la surface des zéros de $f(x)$ coïncide avec $T$.

Ensuite, considérons le cas où $\Psi^{-1}(A)$ ne coïncide pas avec $T$; dans ce cas, $\Psi^{-1}(A)$ se décompose en deux composantes irréductibles dans un voisinage de $z_{0}$; l'une est $T$; et désignons l'autre par $T^{\prime}$; elles sont des composantes irréductibles locales en $z_{0}$ de $\Psi^{-1}(A)$. Soit $h$ une fonction holomorphe sur $\eta$ dans un voisinage de $z_{0}$ qui s'annule identiquement sur $T$ et qui ne s'annule pas identiquement sur $T^{\prime}$; supposons que $h$ soit irréductible en $z_{0}$. D'après la proposition 2 , on peut écrire

$$
h=\psi(x)+\phi(x) \cdot g
$$

où $\psi(x), \phi(x)$ désignent des fonctions holomorphes sur $G$. Puisque $\Psi^{-1}(A)$ se décompose en deux composantes irréductibles $T, T^{\prime}$, on peut écrire d'après la proposition 3

$$
u=v^{2}
$$

où $v$ désigne une fonction holomorphe sur $A$; grâce à W. F. Osgood, on peut écrire

$$
v=\frac{v_{1}^{\prime}}{v_{1}}
$$

où $v_{1}, v_{1}^{\prime}$ sont des fonctions holomorphes sur $G$; on peut supposer qu'elles soient relativement premières en (a). Alors, $v_{1}^{2} D-v_{1}^{\prime 2}$ s'annule identiquement sur $\mathrm{A}$; donc on peut écrire

$$
v_{1}^{2} D-v_{1}^{\prime 2}=\rho f
$$

où $\rho$ est une fonction holomorphe sur $G$ dans un voisinage de (a).

D'autre part, puisque $h$ s'annule identiquement sur $T$, l'une au moins de $\psi-\phi v, \psi+\phi v$ s'annule identiquement sur $A$; supposons par exemple que $\psi-\phi v$ s'annule identiquement sur $A$; par suite, $\psi^{2}-\phi^{2} \cdot u$ s'annule identiquement sur $A$; et donc $\psi^{2}-\phi^{2} \cdot D$ s'annule identiquement sur $A$ aussi; donc on peut écrire

$$
\psi^{2}-\phi^{2} \cdot D=\theta f
$$


où $\theta$ désigne une fonction holomorphe sur $G$ dans un voisinage de (a). Puisque le polynôme de Weierstrass de $h$ est

$$
R(w)=w^{2}-2 \psi(x) \cdot w+\{\psi(x)\}^{2}-\{\phi(x)\}^{2} \cdot D(x),
$$

l'équation de la surface caractéristique $\Psi(Z(h)), Z(h)$ étant la surface des zéros de $h$, est

$$
\{\psi(x)\}^{2}-\{\phi(x)\}^{2} \cdot D(x)=0 .
$$

Donc on peut dire comme suit: Si une surface caractéristique $(B) \theta(x)=0$ coïncide précisément avec $A$ ou ne passe pas par le point $(a), Z(h)$ coïncide précisément avec $T$. Remarquons ici que $v_{1}^{\prime}(a)=0, f(a)=0$; de là l'on a

$$
\theta(a)=-\{\phi(a)\}^{2} \cdot \rho(a) .
$$

Considérons ici une surface caractéristique

$$
\text { ( } \Sigma) \quad \rho(x)=0 \text {; }
$$

$\Sigma$ sera appelée une surface duale de $A$ dans un voisinage de (a). Il $y$ a en général un nombre infini des surfaces duales de $A$, parce que l'on ne peut pas touver $\rho(x)$ d'une manière unique.

THÉORÈME 1. Soit T une surface caractéristique irréductible sur le revêtement $\eta$; supposons qu'elle passe par le point $z_{0}$, et qu'elle soit irréductible en $z_{0}$. Posons $A=\Psi(T)$; et supposons que $\Psi^{-1}(A) \neq T$. Dans ces circonstances, s'il y a une surface duale $(\Sigma) \rho(x)=0$ qui ne passe pas par le point (a), il y a alors une fonction holomorphe $h$ sur $\eta$ dans un voisinage de $z_{0}$ telle que sa surface des zéros coïncide précisément avec $T$.

En effet, posons

$$
\begin{aligned}
& h=v_{1}^{\prime}+v_{1} g \\
& h^{*}=v_{1}^{\prime}-v_{1} g .
\end{aligned}
$$

Calculons $\theta$ pour ces fonctions $h, h^{*}$ :

$$
v_{1}^{\prime 2}-v_{1}^{2} D=-\rho f
$$

donc:

$$
\theta=-\rho,
$$


donc:

$$
\theta(a)=-\rho(a) \neq 0 .
$$

Par suite, la surface caractéristique (B) $\theta(x)=0$ ne passe pas par le point (a); donc, pour la surface des zéros $Z(h)$ de $h$ dans un voisingae de $z_{0}$, on a $\Psi(Z(h))=A$.

Ensuite, supposons que $h\left(\right.$ ou $\left.h^{*}\right)$ s'annule identiquement sur $\Psi^{-1}(A)=$ $T \cup T^{\prime}$; alors $v_{1}^{\prime}$ s'annule identiquement sur $A$. En effet, soit $\left(x^{\prime}\right)$ un point quelconque sur $A$; considérons d'abord le cas où $\Psi^{-1}\left(x^{\prime}\right)$ se compose d'un seul point $r^{\prime} ; r^{\prime}$ est alors un point de ramification de $\eta$; et on a donc $g\left(r^{\prime}\right)=0$; on a donc $h\left(r^{\prime}\right)=v_{1}^{\prime}\left(x^{\prime}\right)+v_{1}\left(x^{\prime}\right) g\left(r^{\prime}\right)=v_{1}^{\prime}\left(x^{\prime}\right)=0$. Ensuite, dans le cas où $\Psi^{-1}\left(x^{\prime}\right)$ se compose des deux points $r_{1}^{\prime}, r_{2}^{\prime}$, on a alors

$$
\begin{aligned}
h\left(r_{1}^{\prime}\right) & =v_{1}^{\prime}\left(x^{\prime}\right)+v_{1}\left(x^{\prime}\right) g\left(r_{1}^{\prime}\right)=0 \\
h\left(r_{2}^{\prime}\right) & =v_{1}^{\prime}\left(x^{\prime}\right)+v_{1}\left(x^{\prime}\right) g\left(r_{2}^{\prime}\right) \\
& =v_{1}^{\prime}\left(x^{\prime}\right)-v_{1}\left(x^{\prime}\right) g\left(r_{1}^{\prime}\right) \\
& =0
\end{aligned}
$$

on a donc $v_{1}^{\prime}\left(x^{\prime}\right)=0$.

Donc l'on a $u \equiv 0$; donc A est une composnate irréductible de $\Psi(S)$, où $S$ désigne la surface critique de $\eta$; donc l'on a $\Psi^{-1}(A)=T$, ce qui est contraire à notre hypothèse. Nous avons ainsi vu que les deux fonctions $h, h^{*}$ ne s'annulent pas identiquement sur $\Psi^{-1}(A)$; donc la surface des zéros de $h$ ou celle de $h^{*}$ coïncide précisément avec $T$.

C.Q.F.D.

2. Surfaecs des zéros des fonctions holomorphes sur la surface de Riemann de la fonction algébrique $\sqrt{D(x, y)}$ où $D(x, y)$ désigne la forme quadratique $D(x, y)=A x^{2}+B x y+C y^{2}\left(B^{2}-4 A C \neq 0\right)$ de deux variables complexes $x, y$.

$1^{\circ}$. Désignons par $\eta=(Z, \Psi)$ la surface de Riemann de $\sqrt{D(x, y)} ; \eta$ est un revêtement analytiquement ramifié à deux feuilles au-dessus de l'espace $C^{2}(x, y)$ de deux variables complexes $x, y$. Traçons dans l'espace $\boldsymbol{C}^{2}(x, y)$ une ligne complexe passant par l'origine $O$

$$
\text { (l) } \quad y=a x
$$


où a est une constante complexe telle que l'on a $A+B a+C a^{2} \neq 0$; cette derniére condition signifie que l'on a $l \neq \Psi(S)$ où $S$ designe la surface critique de $\eta$. Désignons par $u$ la trace de $D(x, y)$ sur $l$; on a alors

$$
u=\left\{\sqrt{A+B a+C a^{2}} \cdot x\right\}^{2} .
$$

Par suite, d'après la proposition 3 , la fibre $\Psi^{-1}(l)$ se décompose en deux composantes irréductibles; désignons-les par $m, m^{\prime}$; et fixons nos yeux sur $m$.

Proposition 5. Il y a une fonction holomorphe $h$ sur $\eta$ telle que sa surface des zéros coïncide précisément avec $m$.

En effet, posons

$$
\begin{aligned}
& p=\frac{2 A+B a}{2 \sqrt{A+B a+C a^{2}}} \\
& q=\frac{B+2 a C}{2 \sqrt{A+B a+C a^{2}}}
\end{aligned}
$$

où l'on prend pour $p, q$ la même racine carrée; et considérons une fonction holomorphe

$$
\psi(x, y)=p x+q y
$$

On a alors

$$
\psi^{2}-D=\frac{\left(B^{2}-4 A C\right)(y-a x)^{2}}{4\left(A+B a+C a^{2}\right)}
$$

donc une surface caractéristique $(\Delta) \psi^{2}-D=0$ coïncide avec $l$. D'autre part, on a sur $l$

$$
\psi=\sqrt{A+B a+C a^{2}} \cdot x ;
$$

par suite, la surface des zéros d'une fonction $h=\psi+\sqrt{D}$ ou celle d'une fonction $h^{*}=\psi-\sqrt{D}$ coïncide précisément avec $m$.

C.Q.F.D.

$2^{\circ}$. Ensuite, nous allons chercher des lignes duales de la ligne complexe (l) $y=a x$. Puisque l'on a $u=\left(A+B a+C a^{2}\right) \cdot x^{2}$, on a

$$
D(x, y)-\left(A+B a+C a^{2}\right) \cdot x^{2}=(y-a x) \cdot\{C y+(B+C a) x\} ;
$$

par suite, lorsque l'on pose 


$$
\rho(x, y)=C y+(B+C a) \cdot x,
$$

une ligne complexe $(\Sigma) \rho(x, y)=0$ est une des lignes duales de $l$.

Pour chercher une ligne duale quelconque de $l$, supposons maintenant que l'on puisse écrire $u=v^{2}$ où $v$ désigne une fonction holomorphe sur $l$; mais dans le cas présent, on peut regarder $v$ comme une fonction holomorphe sur l'espace $C^{2}(x, y)$, parce que $l$ est non-singulière. Alors, puisque $D-v^{2}$ s'annule identiquement sur $l$, elle est divisible par $f=y-a x$; donc on peut écrire

$$
D-v^{2}=\rho_{1} f
$$

où $\rho_{1}$ désigne une fonction holomorphe sur l'espace $C^{2}(x, y)$ dans un voisinage de l'origine $O$. D'autre part, désignons par $m$ l'ideal maximum dans $o$ où $o$ désigne l'anneau local des germes des fonctions holomorphes en l'origine $O$ de l'espace $C^{2}(x, y)$. On a alors $D \in m^{2}$; et puisque l'on a $v(0)=0$, On a $v^{2} \in m^{2}$; donc $D-v^{2} \in m^{2}$. Puisque $f$ est une expression linéaire, on a $f \in m, f \notin m^{2}$; donc, d'après $D-v^{2}=\rho_{1} f_{1}$ on a $\rho_{1} \in m$; à savoir, $\rho_{1}(0,0)=0$.

Nous avons ainsi vu la proposition suivante:

Proposition 6. Toute ligne duale de l passe par l'origine $O$ de l'espace $C^{2}(x, y)$.

Nous avons trouvé dans le théorème 1 une condition suffisante pour qu'une surface caractéristique irréductible sur un revêtement à deux feuilles peut se représenter localement comme l'ensemble des zéros d'une seule fonction holomorphe; mais d'après les propositions 5,6 , on peut conclure que celle-ci n'est pas une condition nécessaire.

3. Deux familles $\{E(s)\}$ et $\{F(s)\}$ des surfaces caractéristiques qui engendrent l'exemple de Grauert-Remmert $\xi=\left(Y, \Phi, C^{3}(v)\right)$.

$1^{\circ}$. Considérons une fonction méromorphe

$$
f=\frac{z_{1}}{w_{1}}=\frac{z_{2}}{w_{2}}
$$

sur le revêtement $\xi$. Le polynôme de Weierstrass de $f$ est

$$
Q(x)=\left(v_{0}-v_{1}-v_{2}\right) x^{2}+\left(v_{1}-v_{2}\right) x-v_{0} ;
$$

le degré de $Q(x)$ est deux; et ceci coïncide avec le nombre des feuilles de $\xi$; donc 
la fonction $f$ a les éléments de Taylor différents pour toute paire de points différents dans $\xi$. Utilizant cette fonction méromorphe $f$, chaque surface caractéristique irréductible $E(s)$ peut se représenter par l'équation $f=s$; en particulier, $E(0)$ est la surface des zéros de $f$; et $E(\infty)$ est la surface des pôles de $f$. Puisque $E(0) \cap$ $E(\infty)=\left\{y_{0}\right\}, y_{0}$ est un point d'indétermination de $f$.

En général, considérons un domaine intérieurement ramifié $\eta$; et soient $z_{0}$ un point de $\eta, h$ une fonction méromorphe sur $\eta$ dans un voisinage de $z_{0}$; dans ces circonstances, si $z_{0}$ est un point d'indétermination de $h, h$ sera dite une fonction engendrant le domaine $\eta$ dans un voisinage de $z_{0}$. Pour tout paramétre $s$ parcourant sur la sphére de Riemann $\boldsymbol{P}(s)$, considérons une surface caractéristique $K(s)$ définie par l'équation $h=s$; on a alors dans un voisinage de $z_{0}$

$$
\begin{gathered}
\eta=\bigcup_{s \in \boldsymbol{P}(s)} K(s) \\
z_{0} \in K(s) \cap K\left(s^{\prime}\right) \quad\left(s \neq s^{\prime}\right) ;
\end{gathered}
$$

la fonction $h$ engendre le revêtement $\eta$ à ce sens. La famille $\{K(s)\}$ composée de toutes les surfaces caractéristiques $K(s)$ sera dite la famille continue de $\eta$ en $z_{0}$ engendrée par $h$. La fonction méromorphe $h$ peut se représenter dans un voisinage de $z_{0}$ comme un quotient de deux fonctions holomorphes $u, v$; si $u, v$ n'ont pas un facteur commun qui s'annule en $z_{0}$, on dit que $u, v$ sont relativement premières en $z_{0}$; et si $u, v$ sont relativement premières en $z_{0}$, la représentation $h=\frac{v}{u}$ sera appelée irréductible en $z_{0}$. Si $z_{0}$ n'est pas un point de ramification, ou si $z_{0}$ est un point de ramification uniformisable, il n'y a qu'une seule représentation irréductible en $z_{0}$ de $h$ comme un quotient de deux fonctions holomorphes; mais, si $z_{0}$ est un point de ramification qui n'est pas uniformisable, en général ce n'est pas cela.

Dans ces circonstances, pour toute représentation irréductible quelconque $h=\frac{v}{u}$ en $z_{0}$ de $h$ comme un quotient de deux fonctions holomorphes $u, v$, supposons que, dans tout voisinage de $z_{0}$, il $y$ ait toujours un point $z$ tel que $u$, $v$ ne sont pas relativement premières en $z$; alors $h$ sera appelée de première espèce. Si $h$ n'est pas de première espèce, $h$ sera appelèe de deuxième espèce.

Dans le cas où $z_{0}$ n'est pas un point de ramification, ou dans le cas où $z_{0}$ est un point de ramification uniformisable, toute fonction engendrant $\eta$ en $z_{0}$ est de deuxième espèce. Mais si $z_{0}$ est un point de ramification qui n'est pas uniformisable, il $y$ a réellement en général une fonction engendrant $\eta_{0}$ en $z_{0}$ de première espèce. 
Par exemple, dans l'exemple de Grauert-Remmert $\xi$, la fonction méromorphe $f$ est une fonction engendrant $\xi$ en $y_{0}$ de première espèce. En effect, considérons une représentation irréductible quelconque $f=\frac{v}{u}$ en $y_{0}$ comme un quotient de deux fonctions holomorphes $u, v$; si $u, v$ sont relativement premières en tout point dans un certain voisinage de $y_{0}$, la surface des zéros de $f$ et celle des pôles de $f$ sont données par les équations $v=0$ et $u=0$ respectivement; mais c'est une contradiction.

C.Q.F.D.

Nous allons formuler brièvement ce que nous avons vu:

Proposition 7. Le revêtement $\xi$ est engendré par la fonction $f$ de première espèce.

$2^{\circ}$. Eh bien, nous avons vu dans No. $1,3^{\circ}$ que la fibre $\Phi^{-1}(\Phi(E(s)))$ se decompose en deux composantes irréductibles; l'une est $E(s)$, et l'autre est $F(s)$; on a ainsi la famille $\{F(s)\}$ composée de toutes les surfaces caractéristiques irréductibles $F(s)$.

Considérons ici en général une fonction méromorphe $h$ sur $\xi$; on suppose que $h$ ait les éléments de Taylor différents pour toute paire de points différents dans $\xi$. Puisque $\xi$ est à deux feuilles, il $y$ a un polynôme de Weierstrass

$$
R(x)=a_{0}(v) \cdot x^{2}+a_{1}(v) \cdot x+a_{2}(v)
$$

où $a_{0}(v), a_{1}(v), a_{2}(v)$ désignent des fonctions holomorphes sur l'espace $C^{3}(v)$, tel que $R(h)$ s'annule identiquement sur $\xi$. Posons $R_{0}(x)=a_{0}(v) \cdot R(x)$; on a alors

$$
R_{0}(x)=\left\{a_{0}(v) \cdot x\right\}^{2}+a_{0}(v) a_{1}(v) \cdot x+a_{0}(v) a_{2}(v),
$$

et on a $R_{0}(h) \equiv 0$ sur $\xi$; ceci désigne que $a_{0}(v) \cdot h$ est holomorphe sur $\xi$. Donc, d'aprés la proposition 2 , on peut ècrire

$$
a_{0}(v) \cdot h=\psi(v)+\phi(v) \cdot u
$$

où $\psi(v), \phi(v)$ désignent des fonctions holomorphes sur l'espace $\boldsymbol{C}^{3}(v)$, et $u$ designe $\sqrt{D(v)}$. Or, il $y$ a deux fonctions méromorphes sur $\xi$ définies par l'équation $R(x)=0$; l'une est évidemment $h$; et l'autre est

$$
h^{*}=\frac{\psi(v)-\phi(v) \cdot u}{a_{0}(v)}
$$

$h^{*}$ sera appelée fonction conjuguée de $h$. 
Définissons ensuite la transformation conjuguée $\Pi$ de $\xi$. Soit $r$ un point quelconque dans $\xi$. Dans le cas où $\Phi^{-1}(\Phi(r))=\{r\}$, on pose $\Pi(r)=r$; et dans le cas où $\Phi^{-1}(\Phi(r))$ se compose en deux points $r, r^{\prime}$, on pose $\Pi(r)=r^{\prime}$. Alors, $\Pi$ est une transformation pseudoconforme biunivoque de $\xi$; et on a $\Pi \circ \Pi=1 ; \Pi$ sera appelée transformation conjuguée de $\xi$.

Traçons dans l'espace $C^{3}(v)$ une surface caractéristique irréductible $A$; et supposons que la fibre $\Phi^{-1}(A)$ se décompose en deux composantes irréductibles; désignons-les par $T, T^{\prime}$. Soit $r$ un point quelconque sur $T$; et on pose $\Phi(r)=(v)$; $\Pi(r)$ est alors un point sur $T^{\prime}$; et on a $u(r)+u(\Pi(r))=0$. On a donc

$$
\begin{aligned}
h(r) & =\psi(v)+\phi(v) \cdot u(r) \\
& =\psi(v)-\phi(v) \cdot u(\Pi(r)) \\
& =h^{*}(\Pi(r)) ;
\end{aligned}
$$

c'est-à-dire, on a $h=h^{*} \circ \Pi$. En particulier, si $T$ se définit par l'équation $h(r)=s$, où $s$ désigne un paramètre qui parcourt sur la sphère de Riemann $\boldsymbol{P}(s)$, on peut dire que $T^{\prime}$ se définit par l'équation $h^{*}(r)=s$.

Désignons maintenant par $g$ une fonction conjuguée de $f$. On a

$$
\begin{aligned}
f & =\frac{-v_{1}+v_{2}+u}{2\left(v_{0}-v_{1}-v_{2}\right)} \\
& =\frac{z_{1}-z_{2}}{w_{1}-w_{2}}=\frac{z_{1}+z_{2}}{w_{1}+w_{2}}
\end{aligned}
$$

où

$$
\begin{aligned}
u & =\sqrt{D(v)} \\
& =\left\{\left(v_{1}-v_{2}\right)^{2}-4 v_{0}\left(v_{1}+v_{2}\right)+4 v_{0}^{2}\right\}^{\frac{1}{2}} \\
& =z_{1}+z_{2}+w_{1}+w_{2} ;
\end{aligned}
$$

on a donc:

$$
\begin{aligned}
g & =\frac{-v_{1}+v_{2}-u}{2\left(v_{0}-v_{1}-v_{2}\right)} \\
& =\frac{w_{1}+w_{2}}{w_{1}-w_{2}}=\frac{z_{1}+z_{2}}{z_{1}-z_{2}}
\end{aligned}
$$

Alors, chaque surface caractéristique $F(s)$ se définit par l'équation $g=s$; cette 
fonction $g$ est une fonction engendrant $\xi$ en $y_{0}$ de première espèce. Enumérons les propriétées de la famille $\{F(s)\}$;

(a) $\xi=\bigcup_{s \in \boldsymbol{P}(s)} F(s)$

(b) $F(s) \cap F\left(s^{\prime}\right)=\left\{y_{0}\right\}\left(s \neq s^{\prime}\right)$

(c) $F(s)$ ne peut pas se représenter comme l'ensemble des zéros d'une seule fonction holomorphe dans un voisinage de $y_{0}$.

$3^{\circ}$. Nous allons étudier les relations entre deux fonctions méromorphes $f$ et $g$ sur $\xi$. Prenons des surfaces caractéristiques quelconques $E(s), F\left(s^{\prime}\right)$ appartenant aux familles $\{E(s)\},\{F(s)\}$ respectivement. Alors, on a la proposition suivante:

Proposition 8. Il n'y a aucune fonction méromorphe sur $\xi$ dans un voisinage de $y_{0}$ telle que sa surface des zéros et sa surface des pôles sont $E(s)$ et $F\left(s^{\prime}\right)$ respectivement.

En effet, supposons qu'il $y$ ait une telle fonction méromorphe; et désignons-la $\operatorname{par} h$. Désignons par $\pi=0$ l'équation minimum de la surface caractéristique $\Phi\left(F\left(s^{\prime}\right)\right)$; et considérons le produit $\pi^{j} \cdot h$ de $h$ par $\pi^{j}$ où $j$ désigne l'ordre de pôle de $h$ le long de $F\left(s^{\prime}\right)$. Si $s=s^{\prime}$, la surface des zéros de $\pi^{j} \cdot h$ est $E(s)$; mais c'est une contradiction. Si $s \neq s^{\prime}$, la surface des zéros de $\pi^{j} \cdot h$ se décompose en deux composantes irréductibles $E(s), E\left(s^{\prime}\right)$. Considérons ici une fonction méromorphe

$$
\rho=\frac{f-s}{f-s^{\prime}}
$$

la surface des zéros de $\rho$ coïncide avec $E(s)$; et la surface des pôles de $\rho$ coïncide avec $E\left(s^{\prime}\right)$. L'ordre de pôle de $\rho$ le long de $E\left(s^{\prime}\right)$ est un; l'ordre de zéro de $\pi^{j} \cdot h$ le long de $E\left(s^{\prime}\right)$ est $j$; donc le produit $\rho^{j} \cdot \pi^{j} \cdot h$ de $\pi^{j} \cdot h$ par $\rho^{j}$ est holomorphe sur $\xi$ dans un voisinage de $y_{0}$; et sa surface des zéros coïncide avec $E(s)$; mais c'est une contradiction.

C.Q.F.D.

La proposition 8 signifie que $E(s), F\left(s^{\prime}\right)$ n'appartiennent pas à la même famille continue en $y_{0}$.

Considérons ici en général un domaine intérieurement ramifié $\eta$, et un point quelconque $z_{0}$ dans $\eta$. Soient $p, q$ des fonctions méromorphes engendrant $\eta$ en $z_{0}$; et soient $E, F$ des surfaces caractéristiques définies par les équations $p=s$, $q=t$ respectivement, où $s, t$ sont des constantes complexes; supposons que $E, F$ 
soient irréductibles, mais d'ailleurs quelconques. Dans ces circonstances, si $E, F$ n'appartiennent pas à la même famille continue en $z_{0}, p, q$ seront appelées indépendantes l'une à l'autre en $z_{0}$. On a vu ainsi la proposition suivante:

Proposition 8'. Il y a deux fonctions méromorphes sur $\xi$ indépendantes l'une à l'autre en $y_{0}$.

On ne peut jamais voir ce phénomène dans des domaines sans point critique intérieur.

$4^{\circ}$. THÉORÈme 2. Considérons une fonction holomorphe $h$ sur $\xi$ dans un voisinage de $y_{0}$, mais d'ailleurs quelconque. On suppose que h soit irréductible en $y_{0}$. Alors, il est impossible que toutes les composantes irréductibles de la surface des zéros de $h$ appartiennent en même temps à la famille continue $\{E(s)\}$ (ou $\{F(s)\}$.

En effet, soient $T_{1}, T_{2}, \ldots, T_{l}$ les composantes irréductibles de la surface des zéros de $h$; et supposons que toutes ces composantes irréductibles appartiennent en même temps à la famille continue $\{E(s)\}$. On peut alors poser que $T_{i}=$ $E\left(s_{i}\right)(i=1,2, \ldots, l)$. Pour tout $i=1,2, \ldots, l$, désignons par $k_{i}$ l'ordre de zéro de $h$ le long de $T_{i}$. Posons ici pour tous $i \neq j$

$$
\rho_{i j}=\frac{f-s_{i}}{f-s_{j}}
$$

alors, la surface des zéros de $\rho_{i j}$ coïncide avec $T_{i}=E\left(s_{i}\right)$; et la surface des pôles de $\rho_{i j}$ coïncide avec $T_{j}=E\left(s_{j}\right)$; et l'ordre de zéro de $\rho_{i j}$ le long de $T_{i}$ est un, et l'ordre de pôle de $\rho_{i j}$ le long de $T_{j}$ est un aussi. Par suite, le poduct

$$
\rho=\rho_{12}^{k_{2}+\cdots+k_{l}} \cdots \cdot \rho_{l-3, l-2}^{k_{l-2}+k_{l-1}+k_{l}} \cdot \rho_{l-2, l-1}^{k_{l-1}+k_{l}} \cdot \rho_{l-1, l}^{k_{l}} \cdot h
$$

est holomorphe dans un voisinage de $y_{0}$; et sa surface des zéros coïncide avec $T_{1}=E\left(s_{1}\right)$; mais c'est une contradiction.

C.Q.F.D.

Le théorème 2 montre que, dans l'exemple de Grauert-Remmert $\xi$, en général les circonstances de distribution des composantes irréductibles d'une fonction holomorphe irréductible en $y_{0}$ ne sont pas arbitraires. Au contraire, dans des domaines sans point critique intérieur, une surface des zéros d'une fonction holomorphe irréductible est toujours irréductible.

Examples. i) Considérons dans le revêtement $\xi$ quatre fonctions holo- 
morphes $z_{1}, z_{2}, w_{1}, w_{2}$. Les polynômes de Weierstrass de ces fonctions sont respectivement

$$
\begin{aligned}
& 2 z_{1}^{2}-\left(2 v_{0}+v_{1}-v_{2}\right) z_{1}+v_{0} v_{1}=0 \\
& 2 z_{2}^{2}-\left(2 v_{0}-v_{1}+v_{2}\right) z_{2}+v_{0} v_{2}=0 \\
& 2 w_{1}^{2}+\left(2 v_{0}-3 v_{1}-v_{2}\right) w_{1}-v_{1}\left(v_{0}-v_{1}-v_{2}\right)=0 \\
& 2 w_{2}^{2}+\left(-2 v_{0}+v_{1}+3 v_{2}\right) w_{2}-v_{2}\left(v_{0}-v_{1}-v_{2}\right)=0 .
\end{aligned}
$$

Toutcs ces fonctions sont irréductibles en $y_{0}$; mais toutes les surfaces des zéros de ces fonctions sont réductibles en $y_{0}$. En génréal, pour une fonction méromorphe $h$, désignons par $Z(h)$ sa surface des zéros; on a alors:

$$
\begin{aligned}
& Z\left(z_{1}\right)=E(0) \cup F(-1) \\
& Z\left(z_{2}\right)=E(0) \cup F(1) \\
& Z\left(w_{1}\right)=E(\infty) \cup F(-1) \\
& Z\left(w_{2}\right)=E(\infty) \cup F(1) .
\end{aligned}
$$

ii) Posons

$$
h=v_{1}-v_{2}-u
$$

où $u=\sqrt{D(v)}$; on a alors:

$$
Z(h)=E(0) \cup F(\infty) .
$$

iii) Posons

$$
h=2 v_{0}-v_{1}-v_{2}-u ;
$$

On a alors

$$
Z(h)=E(1) \cup F(-1) .
$$

iv) Soit $s \neq 0, \pm 1$; et posons

$$
\begin{aligned}
& l(v)=v_{0}-\left(\frac{s}{s+1} v_{1}+\frac{s}{s-1} v_{2}\right) \\
& m(v)=\frac{s-1}{s+1} v_{1}-\frac{s+1}{s-1} v_{2}
\end{aligned}
$$




$$
\rho(v)=v_{0}-\frac{1}{s+1} v_{1}+\frac{1}{s-1} v_{2}
$$

On pose

$$
h=m+u \text {. }
$$

Puisque $m^{2}-D=-4 \rho l$, on a

$$
Z(h)=E(s) \cup F\left(\frac{1}{s}\right)
$$

$5^{\circ}$. Soient $G$ un domaine univalent dans l'espace $\boldsymbol{C}^{n}(x)$ des $n$ variables complexes $x_{1}, x_{2}, \ldots, x_{n}$, et $\eta=(Z, \Psi, G)$ un revêtement analytiquement ramifié de $G$ à deux feuilles. On suppose que, pour un point (a) dans $G$, la fibre $\Psi^{-1}(a)$ se compose d'un seul point de ramification qui n'est pas uniformisable; désignonsle par $z_{0}$. Considérons une surface caractéristique irréductible $T$ sur $\eta$ dans un voisinage de $z_{0}$ qui passe par $z_{0}$.

THÉORÈME 3. Il y a une fonction méromorphe sur $\eta$ dans un voisinage de $z_{0}$ telle que sa surface des zéros coïncide avec $T$, et que son ordre de zéro le long de Test un.

En effet, posons $A=\Psi(T)$; et désignons par $f=0$ l'équation minimum de $A$ dans un voisinage de (a). Considérons d'abord le cas où $\Psi^{-1}(A) \neq T$; alors $\Psi^{-1}(A)$ se décompose en deux composantes irréductibles; l'une est $T$, et désignons par $T^{\prime}$ l'autre composante irréductible. D'après la proposition 1 , il $y$ a une fonction holomorphe $D(x)$ sur $G$ telle que $\eta$ est la surface de Riemann d'une fonction algébroïde $u=\sqrt{D(x)}$ définie par l'équation $w^{2}-D(x)=0$; et d'après la proposition 3, la trace de $D$ sur $A$ est en forme de $v^{2}$ où $v$ désigne une fonction holomorphe sur $A$. Grâce à W. F. Osgood, on peut écrire

$$
v=\frac{r_{2}}{r_{1}}
$$

où $r_{1}, r_{2}$ désignent des fonctions holomorphes sur l'espace $\boldsymbol{C}^{n}(x)$ dans un voisinage de (a); on peut supposer que $r_{1}, r_{2}$ soient relativement premières en (a); donc $r_{1}^{2} D-r_{2}^{2}$ s'annule identiquement sur $A$. Posons ici

$$
\begin{gathered}
h=r_{2}+r_{1} u \\
h^{*}=r_{2}-r_{1} u ;
\end{gathered}
$$


alors, d'après ce que nous avons vu, l'une au moins de deux fonctions $h, h^{*}$ s'annule identiquement sur $T$. Remarquons ici qu'il est impossible que $h, h^{*}$ s'annulent identiquement en même temps sur $T$. En effet, si $h \equiv 0, h^{*} \equiv 0$ sur $T$, alors $r_{1}, r_{2}$ s'annule identiquement sur $A ; r_{1}, r_{2}$ sont donc divisible par $f$; ceci contredit notre supposition que $r_{1}, r_{2}$ soient relativement premières en (a).

Supposons par exemple que $h$ s'annule identiquement sur T. Désignons par $S_{1}, S_{2}, \ldots, S_{l}$ des composantes irréductibles excepté $T$ de la surface des zéros de $h$; et désignons par $S$ la union de toutes $S_{i}(i=1,2, \ldots, l)$. Pour tout $i=1,2, \ldots, l$, désignons par $g_{i}=0$ l'équation minimum d'une surface caractéristique $\Psi\left(S_{i}\right)$; et posons $g=g_{1} \cdots \cdot g_{l}$. Alors, pour un entier positif $m$ suffisamment grand, $T$ coïncide avec une surface des zéros d'une fonction méromorphe

$$
p=\frac{h}{g^{m}}
$$

Désignons de plus par $k$ l'ordre de zéro de $h$ le long de $T$; et posons

$$
q=\frac{h}{f^{k-1}} .
$$

Alors $T$ coïncide avec la surface des zéros de $q$; et l'ordre de zéro de $q$ le long de $T$ est un. Considérons ensuite le cas où $\Psi^{-1}(A)=T$. Si $T$ n'est pas contenue dans la surface critique de $\eta$, elle se définit par l'équation $f=0$, où l'on regarde $f$ comme une fonction holomorphe sur $\eta$. Considérons le cas où $T$ est une des composantes irréductibles de la surface critique de $\eta$. Désignons par $S^{\prime}$ la surface caractéristique composée de toutes les composantes irréductibles excepté $T$-même de la surface critique de $\eta$; et désignons par $g=0$ l'équation minimum de $S^{\prime}$; alors $T$ coincide avec la surface des zéros de $r=\frac{u}{g^{m}}$, où $m$ désigne un entier positif suffisamment grand; et l'ordre de zéro de $r$ le long de $T$ est un.

C.Q.F.D.

Pour la fonction méromorphe $q$ que l'on a trouvée dans le théorème 3 , il est possible que $q$ est holomorphe en $z_{0} ; T$ se représente alors dans un voisinage de $z_{0}$ comme l'ensemble des zéros d'une seule fonction holomorphe sur $\eta$.

Exemple. Considérons l'exemple de Grauert-Remmert $\xi=\left(Y, \Phi, C^{3}(v)\right)$; et traçons dans l'espace $\mathbf{C}^{3}(v)$ une surface caractéristique irréductible

$$
\left(v_{1}-v_{2}\right)^{2}-4 v_{0}\left(v_{1}+v_{2}\right)=0 ;
$$

celle-ci passe par l'origine $O$ de l'espace $C^{3}(v)$. On a $D=4 v_{0}^{2}=\left(2 v_{0}\right)^{2}$ sur $A$; 
donc, d'après la proposition 3, la fibre $\Phi^{-1}(A)$ se décompose en deux composantes irréductibles; désignons-les par $T, T^{\prime}$. Posons

$$
h=2 v_{0}+\sqrt{D(v)}
$$

alors la surface des zéros de $h$ coïncide avec $T$ ou $T^{\prime}$. Si la surface des zéros de $h$ coïncide avec $T$ (ou $T^{\prime}$ ), $T^{\prime}$ (ou $T$ ) coïncide alors avec la surface des zéros d'une fonction holomorphe

$$
h^{*}=2 v_{0}-\sqrt{\overline{D(v)}} .
$$

Dans les circonstances du théorème 3 , supposons que $T$ ne puisse pas se représenter comme l'ensemble des zéros d'aucune seule fonction holomorphe dans un voisinage de $z_{0} ; q$ est alors une fonction engendrant $\eta$ en $z_{0}$ de première espèce. Désignons par $G(s)$ une surface caractéristique définie par l'équation $q=s$ où $s$ est un paramètre parcourant sur la sphère de Riemann $P(s)$; en particulier, $T=G(0)$

Si G(s) est irréductible, elle ne peut pas représenter comme l'ensemble des zéros d'aucune seule fonction holomorphe sur $\eta$ dans un voisinage de $z_{0}$. En effet, supposons que, pour une fonction holomorphe $h$ sur $\eta$ dans un voisinage de $z_{0}, G(s)$ se définisse par l'équation $h=0$; désignons par $l$ l'ordre de zéro de $h$ le long de $G(s)$. D'autre part, désignons par $k$ l'ordre de zéro de $q-s$ le long de $G(s)$; alors l'ordre de pôle de $\frac{1}{q-s}$ le long de $G(s)$ est $k$. La fonction $\left(\frac{q}{q-s}\right)^{l} \cdot h^{k}$ est donc holomorphe en $z_{0}$; et sa surface des zéros coïncide avec $T=G(0)$; mais ceci contredit notre supposition.

C.Q.F.D.

$6^{\circ}$. Dans les circonstances du théorème 3 , supposons que $T$ ne puisse pas se représenter comme l'ensemble des zéros d'aucune seule fonction holomorphe dans un voisinage de $z_{0}$. On a trouvé alors dans le théorème 3 la fonction méromorphe en forme de

$$
q=\frac{h}{g^{m}}
$$

qui s'annule identiquement sur $T$. Désignons par $q^{*}$ la fonction conjuguée de $q$. Désignons par $H(s)$ une surface caractéristique définie par l'équation $q^{*}=$ $s$ où $s$ est un paramètre parcourant sur la sphère de Riemann $P(s)$. On a $\Psi(H(0))=\Psi(G(0))=A ; A$ se définit par l'équation minimum $f=0$. On peut regarder $f$ comme une fonction holomorphe sur $\eta$; alors la surface des zéros de $f$ 
se décompose en deux composantes irréductibles $H(0), G(0)$; et l'ordre de zéro de $f$ le long de $H(0)$ est un; et celui le long de $G(0)$ est un aussi. Supposons maintenant que $H(0)$ se puisse représenter comme l'ensemble des zéros d'une fonction holomorphe $g$; et désignons par $l$ l'ordre de zéro de $g$ le long de $H(0)$; une fonction $\frac{f^{l}}{g}$ est alors holomorphe en $z_{0}$, et sa surface des zéros coïncide avec $T=G(0)$; mais ceci contredit notre supposition.

Donc $H(0)$ ne peut pas se représenter comme l'ensemble des zéros d'une seule fonction holomorphe. Donc, $q^{*}$ est une fonction engendrant $\eta$ en $z_{0}$ de première espèce.

Si $H(s)$ est irréductible en $z_{0}$, il ne peut pas se représenter comme l'ensemble des zéros d'aucune seule fonction holomorphe dans un voisinage de $z_{0}$; la démonstration est même que celle pour $G(s)$.

Les deux fonctions $q, q^{*}$ sont indépendantes l'une à l'autre en $z_{0}$. En effet, si ce n'est pas cela, il $y$ a de certaines composantes irréductibles $G(s), H\left(s^{\prime}\right)$ de $q, q^{*}$ respectivement, et une fonction méromorphe $m$ sur $\eta$ dans un voisinage de $z_{0}$ telles que, dans un voisinage de $z_{0}, G(s)$ coïncide avec la surface des zéros de $m$, et $H\left(s^{\prime}\right)$ coïncide avec celle des pôles de $m$. Désignons par $l$ l'ordre de pôle de $m$ le long de $H(s)$. Désignons par $k$ l'ordre de zéro de $q^{*}-s^{\prime}$ le long de $H\left(s^{\prime}\right)$, et par $k^{\prime}$ l'ordre de zéro de $q^{*}-s$ le long de $H(s)$. Désignons par $r=0$ l'équation minimum de la surface caractéristique $\Psi(G(s))=\Psi(H(s))$. Alors, une fonction

$$
r^{k^{\prime} l} \cdot m^{k} \cdot\left\{\frac{q^{*}-s^{\prime}}{q^{*}-s}\right\}^{l}
$$

est holomorphe en $z_{0}$; et sa surface des zéros coïncide avec $G(s)$; c'est une contradiction.

Nous allons formuler ce que nous avons vu:

THÉORÈME 4. (1) Soit $T$ une surface caractéristique irréductible sur $\eta$ dans un voisinage de $z_{0}$ qui passe par $z_{0}$. On suppose qu'elle ne puisse se représenter comme l'ensemble des zéros d'aucune seule fonction holomorphe dans un voisinage de $z_{0}$. Alors $T$ peut se représenter dans un voisinage de $z_{0}$ comme l'ensemble des zéros d'une certaine fonction $q$ engendrant $\eta$ en $z_{0}$ de première espèce.

(2) Toute surface caractéristique $G(s)$ définie par l'équation $q=s$ ne peut se représenter, si elle est irréductible en $z_{0}$, comme l'ensemble des zéros d'aucune seule fonction holomorphe dans un voisinage de $z_{0}$. 
(3) Il y a une fonction $q^{*}$ engendrant $\eta$ en $z_{0}$ de première espèce telle qu'elle est indépendante de $q$ en $z_{0}$. Une surface caractéristique $H(s)$ définie par l'équation $q^{*}=s$ ne pent se représenter, si elle est irréductible en $z_{0}$, comme l'ensemble des zéros d'aucune fonction holomorphe dans un voisinage de $z_{0}$.

(4) Considérons une fonction holomorphe $h$ sur $\eta$ dans un voisinage de $z_{0}$ telle qu'elle est irréductible en $z_{0}$, et s'annule identiquement sur $T$. Alors, il est impossible que toute composante irréductible en $z_{0}$ de la surface des zéros de $h$ appartient en même temps à la famille continue $\{G(s)\}$.

La démonstration de (4) est même que celle du théorème 2.

\section{Orderes de zéro des fonctions holomorphes sur les domaines intérieurement remifiés.}

Soit $\eta=(Z, \Psi)$ une surface de Riemann d'une fonction algébrique $\sqrt{x y}$ étalée au-dessus de l'espace $\mathbf{C}^{2}(x, y)$ des deux variables complexes $x, y$. Il $y$ a un seul point de ramification $z_{0}$ au-dessus de l'origine $O$ de l'espace $\mathbf{C}^{2}(x, y)$ qui n'est pas uniformisable. Traçons dans l'espace $\mathbf{C}^{2}(x, y)$ une ligne complexe

$$
\text { (l) } y=x
$$

qui passe par l'origine $O$; alors la fibre $\Psi^{-1}(l)$ se décompose en deux composantes irréductibles; designons-les par $E, F$. Dans ces circonstances, d'après la proposition 5, il $y$ a une fonction holomorphe sur $\eta$ dans un voisinage de $z_{0}$ telle que sa surface des zéros coïncide avec $E$; et pour toute telle fonction, son ordre de zéro le long de $E$ est au moins deux.

En effet, prenons une telle fonction holomorphe $h$, mais d'ailleurs quelconque. D'après la proposition 2 , on peut écrire

$$
h=\psi+\phi \cdot \sqrt{x y}
$$

où $\psi, \phi$ désignent des fonctions holomorphes sur l'espace $\mathbf{C}^{2}(x, y)$ dans un voisinage de l'origine $O$. Puisque la surface des zéros de $h$ coïncide avec $E$, on peut écrire

$$
\psi-x \cdot \phi=(y-x) \rho
$$

où $\rho$ désigne une fonction holomorphe sur l'espace $\mathbf{C}^{2}(x, y)$ dans un voisinage de l'origine $O$. On a alors

$$
\psi^{2}-x y \cdot \phi^{2}=\{x \cdot \phi+(y-x) \cdot \rho\}^{2}-x y \cdot \phi^{2}
$$




$$
=(x-y) \cdot\left\{x \cdot \phi^{2}-2 x \cdot \phi \rho+(x-y) \cdot \rho^{2}\right\} .
$$

Posons ici

$$
\pi=x \cdot \phi^{2}-2 x \cdot \phi \rho+(x-y) \cdot \rho^{2} .
$$

Une surface caractéristique (B) $\pi=0$ passe par l'origine $O$ de l'espace $\mathbf{C}^{2}(x, y)$, parce que l'on a $\pi(0,0)=0$. Mais, puisque la surface des zéros de $h$ coïncide avec $E$, il faut que B coïncide avec $l$; par suite, $\pi$ est divisible par $x-y$; et on peut donc écrire

$$
\pi=(x-y) \cdot \mu,
$$

où $\mu$ désigne une fonction holomorphe sur l'espace $\mathbf{C}^{2}(x, y)$ dans un voisinage de l'origine $O$. Donc:

$$
\psi^{2}-x y \cdot \phi^{2}=(x-y)^{2} \cdot \mu
$$

Donc:

$$
h=\psi+\phi \cdot \sqrt{x y}=\frac{\psi^{2}-x y \cdot \phi^{2}}{\psi-\phi \cdot \sqrt{x y}}=\frac{(x-y)^{2} \cdot \mu}{\psi-\phi \sqrt{x y}} .
$$

Ceci montre que l'ordre de zéro de $h$ le long de $E$ est au moins deux.

C.Q.F.D.

Il $y$ a réellement une fonction holomorphe sur $\eta$ telle que sa surface des zéros coïncide avec $E$, et que son ordre de zéro est précisément deux. En effet, posons

$$
h=x+y+2 \sqrt{x y}
$$

cette fonction satisfait aux conditions précitées.

Cet exemple montre que, en général, dans les domaines intérieurement ramifiés, l'ordres de zéro des fonctions holomorphes, telles que ses surfaces des zéros coïncident avec la surface caractéristique donnée, ne sont pas arbitraires.

\section{Bibliographie}

[1] H. Grauert-R. Remmert, Singularitäten komplexen Mannigfaltigkeiten und Riemannsche Gebiete, Math. Z., 67 (1957), 103-128.

[2] H. Grauert-R. Remmert, Komplexer Räume, Math. Ann., 136 (1958), 245-318.

[3] R. KAWAI, On the construction of a holomorphic function in the neighbourhood of a critical point of a ramified domain, Contribution to Function Theory, Tata Institute of Funda- 
mental Research, Bombay (1960).

[4] К. ОкА, Sur les fonctions analytiques de plusieurs variables, VIII. Lemme fondamental, J. Math. Soc. Japan. 3 (1951), 204-214, 259-278. 